\title{
Large-Area, Transfer-Free, Oxide-Assisted Synthesis of Hexagonal Boron Nitride Films and Their Heterostructures with $\mathrm{MoS}_{2}$ and $\mathrm{WS}_{2}$
}

\author{
Sanjay Behura, Phong Nguyen, Songwei Che, Rousan Debbarma, and Vikas Berry* \\ Department of Chemical Engineering, University of Illinois at Chicago, Chicago, Illinois 60607, United States
}

Supporting Information

ABSTRACT: Ultrasmooth hexagonal boron nitride (h-BN) can dramatically enhance the carrier/phonon transport in interfaced transition metal dichalcogenides (TMDs), and amplify the effect of quantum capacitance in field-effect gating. All of the current processes to realize h-BN-based heterostructures involve transfer or exfoliation. Rational chemistries and process techniques are still required to produce large-area, transfer-free, directly grown TMDs/BN heterostructures. Here, we demonstrate a novel boron-oxygen chemistry route for oxide-assisted nucleation and growth of large-area, uniform, and ultrathin h-BN directly on oxidized substrates (B/ $\mathrm{N}$ atomic ratio $=1: 1.16 \pm 0.03$ and optical band gap $=5.51 \mathrm{eV})$. These intimately interfaced, van der Waals heterostructures of $\mathrm{MoS}_{2} / \mathrm{h}-\mathrm{BN}$ and $\mathrm{WS}_{2} / \mathrm{h}$-BN benefit from 6.27-fold reduced roughness of $\mathrm{h}-\mathrm{BN}$ in comparison to $\mathrm{SiO}_{2}$. This leads to reduction in scattering from roughness and charged impurities, and enhanced carrier mobility verified by an increase in electrical conductivity ( 5 times for $\mathrm{MoS}_{2} / \mathrm{h}-\mathrm{BN}$ and 2 times for $\left.\mathrm{WS}_{2} / \mathrm{h}-\mathrm{BN}\right)$. Further, the heterostructures are devoid of wrinkles and adsorbates, which is critical for $2 \mathrm{D}$ nanoelectronics. The versatile process can potentially be extrapolated to realize a variety of heterostructures with complex sandwiched 2D electronic circuitry.

\section{INTRODUCTION}

A large-area, planar, dielectric substrate with ultraflat, smooth surface, lateral continuity, and a structure free of dangling bonds, polymer adsorbates, and surface traps is critical for enhancement of intrinsic characteristics of van der Waals heterostructures for $2 \mathrm{D}$ nanoelectronics and thermionics. ${ }^{1-3}$ The hexagonal boron nitride ( $\mathrm{h}-\mathrm{BN}$ ), a single atom thick twodimensional sheet of $\mathrm{sp}^{2}$ hybridized boron and nitrogen atoms with a honeycomb lattice, has recently received significant attention due to several superior properties, including high thermal conductivity $(2000 \mathrm{~W} / \mathrm{m} \cdot \mathrm{K}),{ }^{4}$ large mechanical strength, ${ }^{5}$ superior optical transparency, ${ }^{6}$ flexible structure, high chemical stability (up to $1000{ }^{\circ} \mathrm{C}$ ), ${ }^{7}$ deep ultraviolet emission, ${ }^{8}$ thinnest tunneling junction, ${ }^{9}$ and high chemical tolerance (for protective coatings). ${ }^{10,11}$ Electronically, h-BN is an insulator with a large direct band gap $(5.97 \mathrm{eV})^{8}$ and a high dielectric constant $(\sim 4) .{ }^{12}$ Several routes have been developed to synthesize h-BN on metal catalytic surfaces via chemical vapor deposition (CVD). ${ }^{13}$ However, growth of h-BN on metal surfaces $\left(\mathrm{Cu},{ }^{13} \mathrm{Ni},{ }^{14} \mathrm{Co},{ }^{15} \mathrm{Pt},{ }^{16}\right.$ and $\mathrm{Cu}-\mathrm{Ni}$ alloy $\left.{ }^{17}\right)$ requires substrate unloading and additional wet/dry transfer processes; for example, h-BN on metal films is supported by a sacrificial polymer layer, followed by metal etching and polymer/h-BN transfer to target substrates. Such transfer process is neither robust, nor reliable and consistently degrades h-BN's structural properties via formation of tears, folds, wrinkles, and adsorption of polymeric impurities. ${ }^{18}$

Here, via leveraging the boron/oxygen bond, we introduce an oxide-assisted growth of direct formation of large-area,

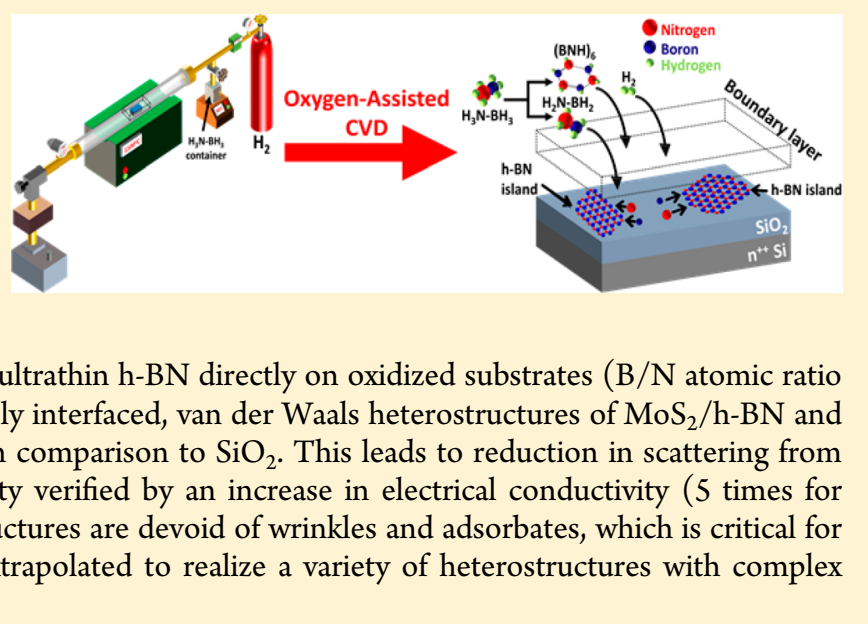

continuous thin films of h-BN on Si-based dielectric $\left(\mathrm{SiO}_{2}\right.$ and quartz) substrates, eliminating the need for metal catalysts, substrate unloading from CVD, postsynthesis transfer steps, and the resultant polymer contamination. Such manufacturability will benefit several electronic, photonic, composite, and mechanical applications. Furthermore, h-BN-modified $\mathrm{SiO}_{2} / \mathrm{Si}$ substrates can be directly applied for realizing van der Waals heterostructures with transition metal dichalcogenides (TMDs) such as $\mathrm{MoS}_{2}$ and $\mathrm{WS}_{2}$. These intimately interfaced, van der Waal heterostructures $\left(\mathrm{MoS}_{2} / \mathrm{h}-\mathrm{BN}\right.$ and $\left.\mathrm{WS}_{2} / \mathrm{h}-\mathrm{BN}\right)$ fabricated through all-CVD growth process (without involving any transfer-related techniques) benefit from 6.27 -fold reduced roughness of h-BN in comparison to Si-based dielectric substrates $\left(\mathrm{SiO}_{2}\right)$. Subsequently, this leads to reduction in surface roughness scattering and charged impurity scattering for the enhancement of intrinsic charge carrier mobility for TMDbased heterostructures. Further, the heterostructures are devoid of surface wrinkles and adsorbates, which is critical for 2D nanoelectronics. Therefore, the versatile process can potentially be extrapolated to realize a variety of heterostructures with welldefined stacking for complex sandwiched 2D electronic circuitry.

\section{EXPERIMENTAL SECTION}

A low-pressure CVD (LPCVD) system was designed (Figure 1a), where the substrate was placed in a split tube furnace with a fused

Received: July 29, 2015

Published: September 21, 2015 


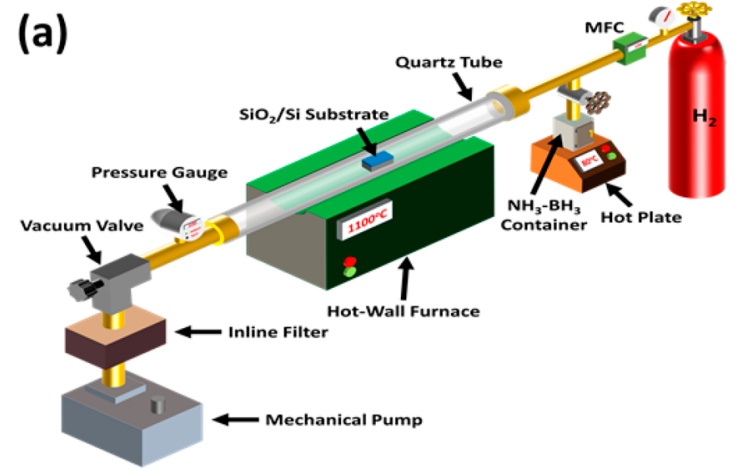

(b)

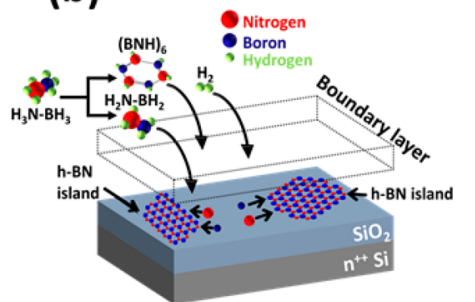

(c)

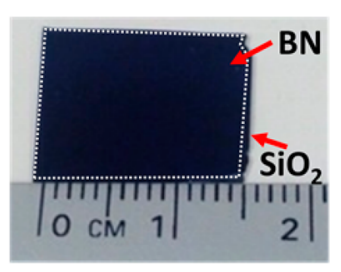

Figure 1. CVD system setup for the growth of h-BN on $\mathrm{SiO}_{2} / \mathrm{Si}$ substrates (thermal conditions are described in Figure S-2), (b) schematic for the proposed growth mechanism, and (c) the h-BN film on $\mathrm{SiO}_{2} / \mathrm{Si}$ of $1.5 \mathrm{~cm} \times 1.5 \mathrm{~cm}$ area.

quartz tube (outside diameter of 1 in.), and a separate heated chamber was employed for h-BN precursors: solid ammonia-borane $(\mathrm{AB})$ $\left(\mathrm{NH}_{3}-\mathrm{BH}_{3}\right)$ complex. The substrates $\left(\mathrm{SiO}_{2} / \mathrm{Si}\right.$ and quartz) were cleaned using piranha solution (a volumetric mixture of $3: 1$ of $98 \%$ $\mathrm{H}_{2} \mathrm{SO}_{4}$ to $35 \% \quad \mathrm{H}_{2} \mathrm{O}_{2}$ ). Furthermore, the surface characteristics (bonding state and degree of wettability) of substrates were analyzed by contact angle measurement (Goniometer, Rame-Hart, Inc.) and Xray photoelectron spectroscopy (XPS, Kratos AXIS-165) (Figure S-1). For h-BN growth, the piranha treated substrates were directly placed in the center of the quartz tube's heating zone and heated to $1100{ }^{\circ} \mathrm{C}$ in $\mathrm{H}_{2}$ atmosphere to restrict further oxidation. After the tube heating zone reached $1100{ }^{\circ} \mathrm{C}$, the $\mathrm{AB}$ was heated at $\sim 100{ }^{\circ} \mathrm{C}$ to thermally decompose into $\mathrm{H}_{2}$ gas, monomeric aminoborane $\left(\mathrm{BH}_{2} \mathrm{NH}_{2}\right)$, and borazine $\left((\mathrm{HBNH})_{3}\right) .{ }^{13}$ The mixture was transported to the chamber containing the substrate via supplied $\mathrm{H}_{2}$ carrier gas. The h-BN synthesis takes place at a pressure of 5-10 Torr, and the reaction time was varied from $10 \mathrm{~s}$ to $60 \mathrm{~min}$ followed by fast cooling $\left(\sim 100{ }^{\circ} \mathrm{C} /\right.$ min). The detailed CVD thermal processing conditions are presented in Figure S-2. The synthesized thin films of h-BN were further characterized by a combined confocal Raman-atomic force microscopy (Raman-AFM, WITEC $\alpha$-300RA with laser wavelength of $532 \mathrm{~nm}$ ), XPS, and transmission electron microscopy (TEM, JEOL JEM-3010). For the AFM measurement of surface roughness and film thickness, the h-BN on $\mathrm{SiO}_{2} / \mathrm{Si}$ surface was patterned through microfabrication etching process: e-beam evaporation (Varian), UV-photolithography (Karl Suss MA6), and reactive ion etching (RIE, Oxford Instruments).

\section{RESULTS AND DISCUSSION}

On the basis of the spectroscopic results (discussed later), we determined that the h-BN's lateral growth occurs via nucleation of $\mathrm{B}-\mathrm{O}$ bonds formed post borazane's thermal decomposition (to form its intermediates, i.e., $\mathrm{BH}_{2} \mathrm{NH}_{2}$ and aminoborane). The formation mechanism of the h-BN films on $\mathrm{SiO}_{2} / \mathrm{Si}$ substrates can be understood via the vapor-solid-solid growth mechanism. ${ }^{19,20}$ It is known that for carbon nanotube and graphene synthesis, the oxygen-aided growth is a surface deposition process with a very low reaction rate. ${ }^{19,20}$ CVD growth of graphene is influenced by kinetic factors: the relative magnitude of the mass transport coefficients $\left(h_{\mathrm{g}}\right)$ and surface reaction constants $\left(K_{\mathrm{s}}\right) .^{20,21}$ The growth of thickness uniformity for h-BN film occurs when $h_{\mathrm{g}} \gg K_{\mathrm{s}}$ (surface reaction controlled region), while the growth of thickness nonuniform film occurs when $h_{\mathrm{g}} \ll K_{\mathrm{s}}$ (mass transport limited region). The mass transport coefficient $\left(h_{\mathrm{g}}\right)$ can be defined as the ratio of diffusivity $\left(D_{\mathrm{g}}\right)$ and the thickness of boundary layer $(\delta): h_{\mathrm{g}}=D_{\mathrm{g}} / \delta$ (see the Supporting Information). At low pressure, the collision of the active precursors is less, leading to a higher diffusivity $\left(D_{\mathrm{g}} \propto 1 /\right.$ (total pressure $\left.)\right)$. Despite the increase in the thickness of the boundary layer at low pressure, the increase of $D_{\mathrm{g}}$ is much higher. Consequently, the mass transport coefficient $h_{\mathrm{g}}$ is high at low pressure, leading to improved diffusion of the precursors through the boundary, making surface reaction the rate-limiting step. Furthermore, the $\mathrm{SiO}_{2} / \mathrm{Si}$ surface is suspected to possess a relatively low value of $\mathrm{K}_{\mathrm{s}}{ }^{20}$ which may enable large-area uniform growth of h-BN sheet. The grain size for h-BN is found to be of $10-20 \mu \mathrm{m}$ and is presented in Figure S-3 via growth of lateral heterostructures of h-BN (incompletely grown) and graphene. The schematic for the h-BN growth on $\mathrm{SiO}_{2} / \mathrm{Si}$ surfaces is presented in Figure $1 \mathrm{~b}$, and an optical micrograph of $\mathrm{h}-\mathrm{BN} / \mathrm{SiO}_{2} / \mathrm{Si}$ is also presented in Figure 1c.

Consistent with our theory, Tay et al. ${ }^{22}$ explain that surface oxygen in $\mathrm{Cu}$ favors the h-BN growth as it facilitates dissociation of the precursor gas and enhances the dehydrogenation process along the edges of the h-BN domains. The growth of $\mathrm{h}-\mathrm{BN}$ in the present case can be described by the following elementary steps: (1) At $100{ }^{\circ} \mathrm{C}$, the solid $\mathrm{AB}$ complex is sublimed into the gaseous phase and carried into the reaction zone by $\mathrm{H}_{2}$ gas. (2) Before reaching the $1100{ }^{\circ} \mathrm{C}$ zone, the $\mathrm{AB}$ complex is thermally decomposed into $\mathrm{H}_{2}, \mathrm{BH}_{2} \mathrm{NH}_{2}$, and $(\mathrm{HBNH})_{3}$. (3) At a temperature of $1100{ }^{\circ} \mathrm{C}$, borazine $\left(\mathrm{B}_{3} \mathrm{H}_{6} \mathrm{~N}_{3}\right)$ and aminoborane $\left(\mathrm{BH}_{2} \mathrm{NH}_{2}\right)$ molecules dissociate into boron and nitrogen radicals, which may diffuse along the $\mathrm{SiO}_{2}$ surface. (4) Boron radical starts the nucleation of a h-BN domain by binding at the oxygen dangling bond of $\mathrm{SiO}_{2}$. (5) If the rate of attachment of boron radicals and nitrogen radicals at the edge of h-BN domain is higher than the rate of their detachments, then the h-BN domains start to expand into continuous h-BN sheet. The oxygen-assisted nucleation of h$\mathrm{BN}$ film is also apparent from the XPS spectrum for B 1s, which is described later. The edge-attachment and lattice integration involve dehydrogenation at domain edges; the edge-attachment barrier is effectively reduced due to the strong binding energy between $\mathrm{B}$ and $\mathrm{O}$. Further, experiments without thermally grown $\mathrm{SiO}_{2}$ and only silicon $(\mathrm{Si})$ show no coverage of h-BN, indicating the importance of oxide groups. The Raman spectrum and optical microscopic image for the post h-BN growth Si surface is discussed in Figure S-4.

The quality and uniformity of h-BN film formed via oxideassisted LPCVD on $\mathrm{SiO}_{2} / \mathrm{Si}$ substrates are analyzed by Raman spectroscopy. In this case, the growth is carried out for $1 \mathrm{~min}$. Figure 2a shows the optical microscopic image indicating the uniform formation of h-BN with subsequent Raman peaks at $\sim 1373-1375 \mathrm{~cm}^{-1}$ corresponding to $\mathrm{E}_{2 \mathrm{~g}}$ phonon vibration represented in Figure $2 \mathrm{~b}$. The Raman spectral position mapping is presented in Figure $2 \mathrm{c}$ with the regions marked with blue, red, and black circles corresponding to Raman spectra in Figure $2 \mathrm{~b}$. The homogeneous color contrast (Figure $2 \mathrm{a}$ and c) clearly shows a continuous and uniform h-BN film formation on $\mathrm{SiO}_{2} / \mathrm{Si}$ substrates. The weak Raman intensity at $\sim 1373-1375 \mathrm{~cm}^{-1}$ corresponds to the thin h-BN films. This is 

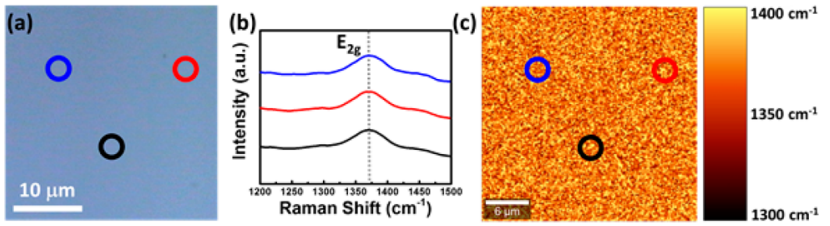

(d)

(e)
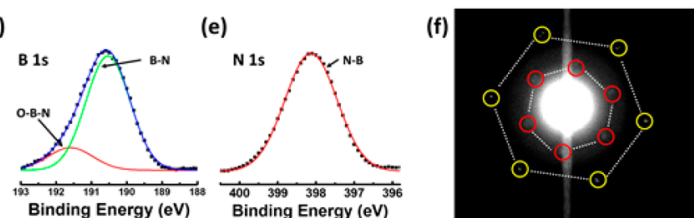

Figure 2. Raman spectroscopic analysis of h-BN films on $\mathrm{SiO}_{2} / \mathrm{Si}$ surfaces. (a) The optical microscopy image, (b) $\mathrm{E}_{2 \mathrm{~g}}$ peak centered at 1373-1375 $\mathrm{cm}^{-1}$, and (c) scanning Raman position mapping of $\mathrm{E}_{2 \mathrm{~g}}$ peak. The colored circles at different areas of (a) and (c) correspond to the Raman spectra in (b). XPS spectroscopic analysis for (d) B 1s, (e) $\mathrm{N} \mathrm{1s}$, and (f) SAED of h-BN film transferred onto TEM grids.

to note that under compressive or tensile stress, the Raman peak shifts to higher or lower frequency, respectively. ${ }^{23}$ We also observe that the Raman $\mathrm{E}_{2 \mathrm{~g}}$ peak position shifts to higher frequency, which may be attributed to the formation of surfaceinduced stress in the h-BN films. ${ }^{24}$

The growth mechanism is analyzed via the bonding state and the elemental composition of boron (B) and nitrogen $(\mathrm{N})$, which is obtained from XPS. The B 1s spectrum demonstrates two peaks fitted with Gaussian components (full width at halfmaximum, fwhm $\sim 1.5 \mathrm{eV}$ ). The peaks at binding energy (BE) $\sim 190.53$ and $\sim 191.59 \mathrm{eV}$ for the $\mathrm{B} 1 \mathrm{~s}$ (Figure $2 \mathrm{~d}$ ) are attributed to $\mathrm{B}-\mathrm{N}^{25,26}$ and $\mathrm{O}-\mathrm{B}-\mathrm{N}^{27,28}$ bonding, respectively. Figure 2e represents the $\mathrm{N} 1 \mathrm{~s}$ peak at $\mathrm{BE} \sim 398.13 \mathrm{eV}$ for the $\mathrm{N}-\mathrm{B}$ bonding. ${ }^{25,26}$ The different components contributing to the spectra are deconvoluted by a curve-fitting procedure. This also confirms that the oxygen $(\mathrm{O})$ works as $\mathrm{B}$ nucleation sites, which continues to connect to $\mathrm{N}$ to form a uniform h-BN film. It is important to note that there is no N-O peak in the XPS spectra for $\mathrm{N} \mathrm{1s}$, implying that $\mathrm{N}$ binds only with $\mathrm{B}$, and not with $\mathrm{O}$ on $\mathrm{SiO}_{2}$. Further, the $\mathrm{B}-\mathrm{O}$ peak also indicates the presence of boron terminated edges bonded to $\mathrm{SiO}_{2}$, where $\mathrm{B}$ is bound to $\mathrm{O}$ and possibly two N's. In addition, the XPS of $\mathrm{O}$ in the h-BN film further confirms the bonding states of $\mathrm{B}$ and $\mathrm{O}$ (Figure S-5a). The survey scan for the identification of all of the elemental composition in the h-BN film is presented in Figure $\mathrm{S}-5 \mathrm{~b}$. The elemental stoichiometric analysis confirms an almost equal composition of $\mathrm{B}$ and $\mathrm{N}$ elements: $\mathrm{B} / \mathrm{N}$ ratio $=1: 1.16 \pm$ 0.03 . This result in combination with the selected area electron diffraction (SAED) pattern (Figure 2f) confirms the hexagonal lattice in the synthesized film. Further confirmation is presented through the XRD pattern of h-BN films on $\mathrm{SiO}_{2} / \mathrm{Si}$ surfaces and described in Figure S-5c.

Figure 3 a demonstrates the AFM of patterned h-BN with the inset showing the line-scan profile of $\mathrm{h}$ - $\mathrm{BN}$ films indicating thickness $\sim 7 \mathrm{~nm}$. Figure $3 \mathrm{~b}$ presents the surface characteristics for $\mathrm{SiO}_{2}$ and h-BN-modified $\mathrm{SiO}_{2}$. Figure $3 \mathrm{c}$ depicts the roughness histograms fitted by Gaussian distributions with standard deviations of $1.37 \mathrm{~nm}$ for the h-BN-modified $\mathrm{SiO}_{2}$ surface and $8.59 \mathrm{~nm}$ for the $\mathrm{SiO}_{2}$ surface. The h-BN consists of planar $\mathrm{sp}^{2}$ hybridized bonds and weak out-of-plane van der Waals bonds, which lead to a decreased density of absorbed impurities and smooth surface. In addition, because of its high energy surface optical phonon modes, a reduced scattering from electron-phonon interactions is expected at the TMDs/
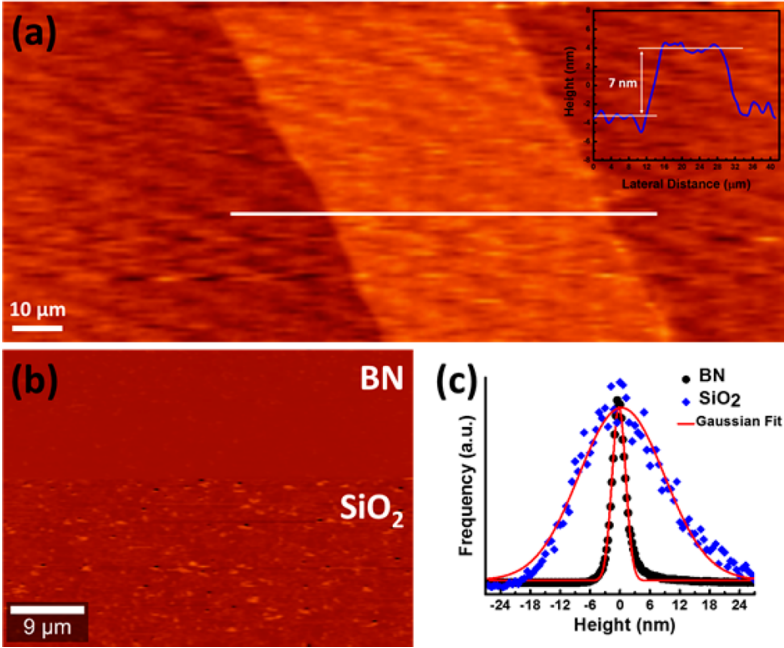

Figure 3. AFM analysis of (a) patterned h-BN with line scan in the inset showing thickness $<7 \mathrm{~nm}$, (b) surface roughness of $\mathrm{SiO}_{2} / \mathrm{Si}$ before and after h-BN growth, and (c) roughness histogram of the height distribution measured via $\mathrm{AFM}$ for $\mathrm{SiO}_{2} / \mathrm{Si}$ (blue ) and h-BN

(O) with Gaussian fit to the distribution presented in red solid lines.

h-BN heterostructure interface. The thickness of h-BN film is also controlled by adjusting the reaction time $(10 \mathrm{~s}, 1 \mathrm{~min}$, and $5 \mathrm{~min}$ ). While the $10 \mathrm{~s}$ growth resulted in h-BN with Raman signature of a monolayer, the 1 and 5 min growth reproduced 7 and $20 \mathrm{~nm}$ of the h-BN platform, respectively, as shown and discussed in the Figure S-6. We envision that the growth rate of this newly developed oxide-assisted growth can be achieved by engineering the catalytic parameters (reaction time, surface oxide concentration, and reaction temperature).

To further confirm the oxide-assisted growth mechanism, we have synthesized large-area h-BN film directly onto a quartz slide via the same CVD process as performed for $\mathrm{SiO}_{2} / \mathrm{Si}$ substrates. As expected, we found the h-BN films on the post CVD quartz substrates $(2 \mathrm{~cm} \times 1.5 \mathrm{~cm}$ area $)$ as shown in Figure 4a with the corresponding Raman peak centered at 1375 $\mathrm{cm}^{-1}$ (Figure 4b). Subsequently, the UV-visible absorption
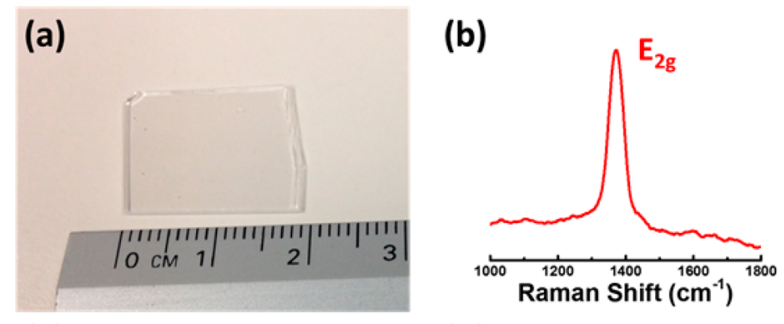

(c)

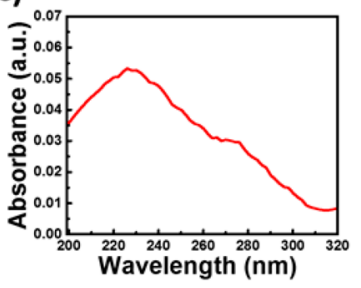

(d)

Figure 4. Direct CVD growth of h-BN on quartz surfaces. (a) Camera view of $2 \mathrm{~cm} \times 1.5 \mathrm{~cm}$ area. (b) Raman spectrum of h-BN films, (c) room temperature UV-vis absorbance spectra, and (d) Tauc plot corresponds to the optical band gap of $5.51 \mathrm{eV}$. 
spectrum is carried out for probing the h-BN's optical energy gap based on its optically induced direct-band transition. As the h-BN film is directly on the quartz surface, the quartz background is subtracted using a blank quartz. The following Tauc's equation was used to determine the optical band gap $E_{\mathrm{g}}{ }^{29}$

$$
\alpha h \nu=A\left(h \nu-E_{g}\right)^{1 / 2}
$$

where $\alpha$ is the optical absorption coefficient, $h \nu$ is the energy of incident photon, $A$ is the proportionality constant, and $E_{\mathrm{g}}$ is the optical energy band gap. Figure $4 c$ shows the absorption spectrum, which displays one sharp absorption peak at $225 \mathrm{~nm}$. On the basis of Tauc's formulation in eq 1, it is speculated that the plot of $(\alpha h \nu)^{2}$ versus $h \nu$ should be a straight line at the absorption range, and the intersection point with the $h \nu$-axis is the optical energy band gap, $E_{\mathrm{g}}$. Figure $4 \mathrm{~d}$ shows the $(\alpha h \nu)^{2}$ versus $h \nu$ curve acquired from the thin h-BN film on quartz surface. The calculated optical band gap is $5.51 \mathrm{eV}$. The theoretical calculations predict the energy band gap for singlelayer h-BN film to be $6 \mathrm{eV}$ because the equivalent bands do not cross each other. ${ }^{30}$ Considering multilayer structure in our h$\mathrm{BN}$ films, the measured gap is smaller than the theoretical gap value of a single-layer, due to the layer-layer interaction increasing the dispersion of the electronic bands. However, the band gap is larger than that of bulk h-BN $(5.2-5.4 \mathrm{eV})$; therefore, the process produces thin $\mathrm{BN}$ films. ${ }^{31}$

To leverage the potential of the direct grown h-BN film, we next fabricated heterostructured films with $\mathrm{MoS}_{2}$ and $\mathrm{WS}_{2}$. Recent reports indicate dramatic improvement in optical and electronic properties of $\mathrm{TMD}^{32,33}$ layers stacked on h-BN dielectrics. ${ }^{34,35}$ A substantial amount of previous work reports on mechanical exfoliation of at least one of the TMDs or h-BN materials to form both the lateral and the vertical heterostructures. ${ }^{34,36,37}$ However, a direct and transfer-free allCVD process for the fabrication of high-quality $\mathrm{MoS}_{2}: \mathrm{h}-\mathrm{BN}$ and $\mathrm{WS}_{2}$ :h-BN vertical heterostructured films has not been reported. Here, we show fabrication of all-direct, CVD-growth of both $\mathrm{MoS}_{2} / \mathrm{h}-\mathrm{BN}$ and $\mathrm{WS}_{2} / \mathrm{h}-\mathrm{BN}$ vertical heterostructures and outline their structural, optical, and electrical properties. The CVD synthesis methods of $\mathrm{MoS}_{2}$ and $\mathrm{WS}_{2}$ on h-BN films are discussed in the Supporting Information with the CVD setup schematic shown in Figure S-7.

Raman spectroscopy is a commanding technique to determine the presence of $\mathrm{MoS}_{2}$ and $\mathrm{WS}_{2}$ films and the number of corresponding layers through the measurement of $\mathrm{E}_{2 \mathrm{~g}}^{\prime}$ and $\mathrm{A}_{1 \mathrm{~g}}$ peak positions and their frequency difference. ${ }^{38}$ The $E_{2 g}^{\prime}$ mode represents the in-plane vibration ( $S$ atoms in opposite direction to the $\mathrm{Mo} / \mathrm{W}$ atom), while the $\mathrm{A}_{1 \mathrm{~g}}$ mode is related to the out-of-plane vibration ( $S$ atoms in opposite directions) (inset of Figure 5a and d). ${ }^{39}$ In Figure 5a and d, $\mathrm{E}_{2 \mathrm{~g}}^{\prime}$ and $A_{1 g}$ peaks of $M_{2}$ grown on $h-B N$ are centered at $\sim 375.6$ and $\sim 401.5 \mathrm{~cm}^{-1}$, respectively, and $\mathrm{E}_{2 \mathrm{~g}}^{\prime}$ and $\mathrm{A}_{1 \mathrm{~g}}$ peaks of $\mathrm{WS}_{2}$ grown on h-BN are centered at $\sim 345$ and $\sim 410.65 \mathrm{~cm}^{-1}$, respectively. The Raman spatial scans (Figures S-8 and S-9) show that the intensity of the h-BN peak $\left(1373-1375 \mathrm{~cm}^{-1}\right)$ corresponds very well with the $\mathrm{MoS}_{2}$ and $\mathrm{WS}_{2}$ peak intensities. Because the peak intensity is sensitive to the distance from the focal plane of the laser, this implies that the general distance between h-BN and TMD layers is consistent and thus governed by van der Waals interaction. Further, the h-BN peak positions do not change after synthesis, which implies that the layers are expected to be electronically isolated.
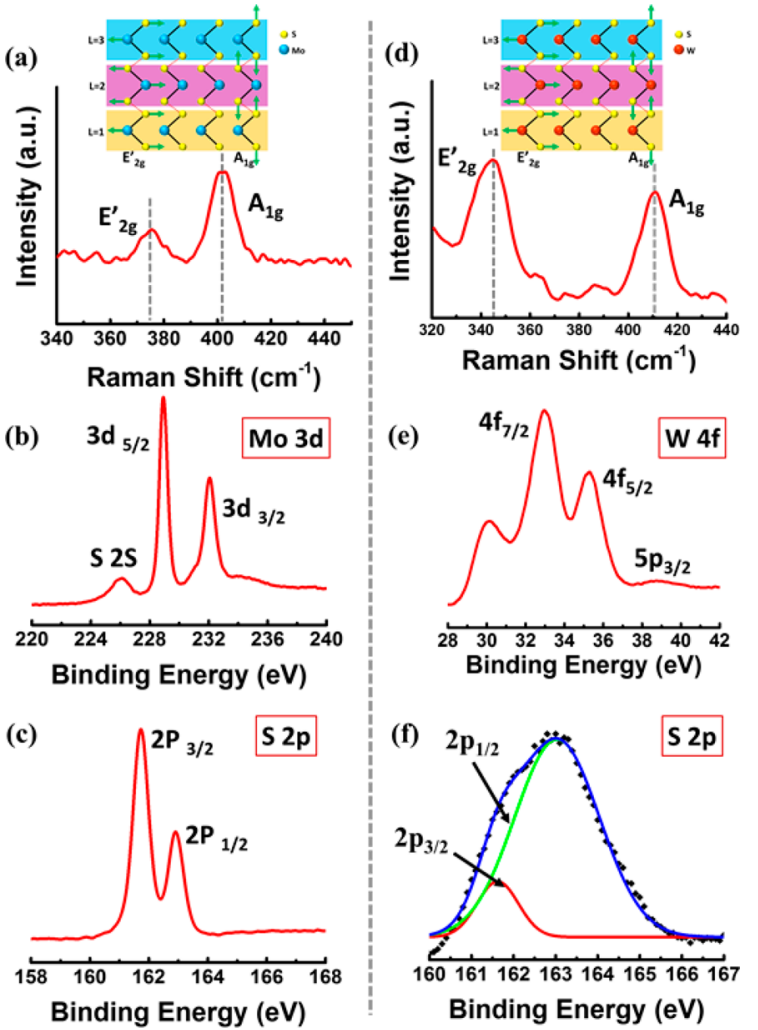

Figure 5. $\mathrm{MoS}_{2}: \mathrm{h}-\mathrm{BN}$ heterostructure: $(\mathrm{a}-\mathrm{c})$ Raman spectrum corresponds to $E_{2 g}^{\prime}$ and $A_{1 g}$ peaks, XPS peaks for Mo 3d and $S$ 2p. $\mathrm{WS}_{2}$ : h-BN heterostructure: (d-f) Raman spectrum corresponds to $\mathrm{E}_{2 \mathrm{~g}}^{\prime}$ and $\mathrm{A}_{1 \mathrm{~g}}$ peaks, XPS peaks for $\mathrm{W} 4 \mathrm{f}$ and $\mathrm{S} 2 \mathrm{p}$. In the inset, Raman vibrational modes for $\mathrm{MoS}_{2}$ in-plane $\mathrm{E}_{2 \mathrm{~g}}^{\prime}$ and out-plane $\mathrm{A}_{1 \mathrm{~g}}$ modes (a) and $\mathrm{WS}_{2}$ in-plane $\mathrm{E}_{2 \mathrm{~g}}^{\prime}$ and out-plane $\mathrm{A}_{1 \mathrm{~g}}$ modes (d).

The chemical composition of $\mathrm{MoS}_{2}$ and $\mathrm{WS}_{2}$ on h-BN is confirmed via XPS. Figure $5 \mathrm{~b}$ shows the Mo $3 \mathrm{~d}$ peaks at 229 and $232 \mathrm{eV}$ correspond to $3 \mathrm{~d}_{5 / 2}$ and $3 \mathrm{~d}_{3 / 2}$, respectively, whereas the $S 2 p$ peak appears at $226 \mathrm{eV}$. The $S 2 p_{3 / 2}$ and $S 2 p_{1 / 2}$ peaks appear at 161.5 and $163 \mathrm{eV}$, respectively, as displayed in Figure 5c. These are consistent with the peak positions from the literature, ${ }^{40}$ again suggesting the electronic isolation from the underlying h-BN sheet. This is also attributed to the thermal stability of h-BN during the growth process. Figure 5e reveals the $\mathrm{W} 4 \mathrm{f}$ peaks at 33 and $35.3 \mathrm{eV}$ correspond to $4 \mathrm{f}_{7 / 2}$ and $4 \mathrm{f}_{5 / 2}$, respectively, whereas the $\mathrm{W} 5 \mathrm{p}_{3 / 2}$ peak appears at $38.8 \mathrm{eV}$. The fitting results for $S 2 p_{3 / 2}$ and $S 2 p_{1 / 2}$ peaks for $W_{2}$ appear at 161.6 and $163 \mathrm{eV}$, respectively (Figure 5f).

The direct growth of heterostructured films has several advantages over the transfer techniques such as the possibility for scaling up for large-area production, and elimination of interfacial contamination. The photoluminescence (PL) of direct growth TMDs on h-BN was investigated by using 532 $\mathrm{nm}$ laser excitation. In Figure $6 \mathrm{a}$, two pronounced PL peaks were located at $1.82 \mathrm{eV}(\mathrm{A})$ and $1.96 \mathrm{eV}(\mathrm{B})$, which are in good agreement with the previous report. ${ }^{36}$ The A peak corresponds to neutral exciton emission from the direct transition at the $\mathrm{K}$ point, and the $\mathrm{B}$ peak is due to the exciton emission from another direct transition between the conduction and valence band.

The influence of h-BN on the electrical property of $\mathrm{MoS}_{2}$ and $\mathrm{WS}_{2}$ is characterized through current-voltage $(I-V)$ measurements. The device fabrication process is as follows: after the synthesis of $\mathrm{TMDs}$ on $\mathrm{SiO}_{2} / \mathrm{Si}$ or quartz substrates 

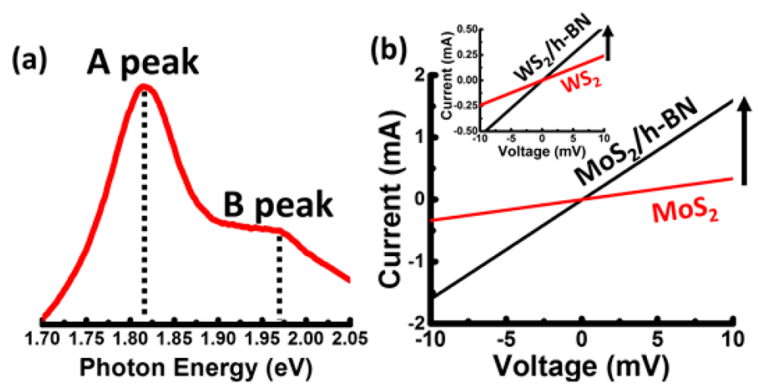

Figure 6. (a) PL spectrum of the direct grown $\mathrm{MoS}_{2}$ on h-BN. I-V characteristics of (b) $\mathrm{MoS}_{2} / \mathrm{h}$ - $\mathrm{BN}$ versus $\mathrm{MoS}_{2} / \mathrm{SiO}_{2}$ and (inset) $\mathrm{WS}_{2} /$ h-BN versus $\mathrm{WS}_{2} / \mathrm{SiO}_{2}$ devices.

with and without h-BN, metal contact electrodes $(\mathrm{Cr}(15 \mathrm{~nm}) /$ $\mathrm{Au}(95 \mathrm{~nm})$ ) were deposited using e-beam evaporator. Positive photoresist was spun onto the film, and photolithography was performed to create the pattern. Figure $6 \mathrm{~b}$ and its inset show the $I-V$ characteristics of the $\mathrm{MoS}_{2} / \mathrm{h}-\mathrm{BN}$ versus $\mathrm{MoS}_{2} / \mathrm{SiO}_{2}$ and $\mathrm{WS}_{2} / \mathrm{h}-\mathrm{BN}$ versus $\mathrm{WS}_{2} / \mathrm{SiO}_{2}$ devices, respectively. The conductivity can be expressed as $\sigma=q \mu_{\mathrm{p}} p+q \mu_{\mathrm{n}} n$, where $q$ is the elementary charge, $\mu_{\mathrm{p}}, \mu_{\mathrm{n}}$ are hole and electron mobilities, and $p, n$ are hole and electron concentrations. Because these TMDs are n-type due to the pinning of the conduction band close to the Fermi level of metal contacts, this equation becomes $\sigma=q \mu_{\mathrm{n}} n$. Furthermore, when the $\mathrm{MoS}_{2}$ and $\mathrm{WS}_{2}$ layers are placed on $\mathrm{SiO}_{2}$ surfaces, these films are unintentionally negatively doped due to the high level of trapped donors. ${ }^{41,42}$ Hence, the electron concentration $(n)$ in $\mathrm{MoS}_{2}$ and $\mathrm{WS}_{2}$ for $\mathrm{TMDs} / \mathrm{SiO}_{2}$ heterostructure is expected to be higher. On the other hand, placing $\mathrm{MoS}_{2}$ and $\mathrm{WS}_{2}$ layers on h$\mathrm{BN}$ surfaces provides an electronically clean platform with limited doping as h-BN lacks trap charges. However, our results show that the currents $(I)$ of $\mathrm{MoS}_{2} / \mathrm{h}-\mathrm{BN}$ and $\mathrm{WS}_{2} / \mathrm{h}-\mathrm{BN}$ are 5and 2-fold higher than $\mathrm{MoS}_{2} / \mathrm{SiO}_{2}$ and $\mathrm{WS}_{2} / \mathrm{SiO}_{2}$, respectively. Because the electron density on $\mathrm{h}-\mathrm{BN}$ is expected to reduce, the increase in total conductivity of TMDs/h-BN implies enhanced electron mobility $\left(\mu_{\mathrm{n}}\right)$, attributed to the smooth h-BN substrate and reduced charged impurity scattering. This conclusively indicates that h-BN enhances the carrier/phonon transport in the interfaced TMDs, and also potentially in graphene as shown in previous work. ${ }^{3}$

\section{SUMMARY AND CONCLUSIONS}

We demonstrate a facile and robust method to grow uniform, large-scale, controllable thickness of h-BN films on $\mathrm{SiO}_{2}$ and quartz substrates. We also provide the details of the growth mechanism, where the oxygen on these surfaces binds with boron to produce nucleation sites for oxide-assisted growth of large-area and continuous films of h-BN. The nucleation density and domain sizes are highly dependent on the surface morphology and oxygen content. In addition, due to the increased surface oxygen on the $\mathrm{SiO}_{2} / \mathrm{Si}$ and the strong binding between $\mathrm{B}$ and $\mathrm{O}$, the edge-attachment energy barrier is reduced. Further, the large-area, van der Waals bound, and electronically isolated TMD heterostructures with h-BN were also grown. The grown h-BN has consistently exhibited its use for enhancing carrier mobility for electronic $2 \mathrm{D}$ nanomaterials by bringing electronic-isolation and providing ultrasmoothness platform. The results from this work provide an avenue for large-area, scalable, directly grown, contamination free, transfer/unloading free, and heterostructure-ready h-BN, which is vital for high-performance 2D nanoelectronics, optoelectronics, and nanothermionics, currently limited by physically exfoliated h-BN flakes or transferred h-BN. Further, the atomically precise placement achieved here can be extended to produce complex sandwiched and lateral architectures for ultrathin integrated 2D-circuitries.

\section{ASSOCIATED CONTENT}

Supporting Information

The Supporting Information is available free of charge on the ACS Publications website at DOI: 10.1021/jacs.5b07739.

Experimental procedures and additional figures (PDF)

\section{AUTHOR INFORMATION}

\section{Corresponding Author}

*vikasb@uic.edu

\section{Notes}

The authors declare no competing financial interest.

\section{ACKNOWLEDGMENTS}

V.B. acknowledges financial support from the National Science Foundation (grants CMMI-1503681 and CMMI-1030963) and the University of Illinois at Chicago. This work made use of instruments in the Electron Microscopy Service (Research Resources Center, UIC). S.B. acknowledges Donovan Briggs, Shikai Deng, and Leonardo Marques for the valuable discussions on $\mathrm{MoS}_{2}$ and $\mathrm{WS}_{2}$ growth. V.B. and S.B. thank Prof. Christos G. Takoudis and Mr. Arghya Bishal for help with the contact angle measurements.

\section{REFERENCES}

(1) Geim, A. K.; Grigorieva, I. V. Nature 2013, 499, 419.

(2) Qi, Z. J.; Hong, S. J.; Rodríguez-Manzo, J. A.; Kybert, N. J.; Gudibande, R.; Drndić, M.; Park, Y. W.; Johnson, A. T. C. Small 2015, $11,1402$.

(3) Dean, C. R.; Young, A. F.; MericI; LeeC; WangL; SorgenfreiS; WatanabeK; TaniguchiT; KimP; Shepard, K. L.; Hone, J. Nat. Nanotechnol. 2010, 5, 722.

(4) Tao, O.; Yuanping, C.; Yuee, X.; Kaike, Y.; Zhigang, B.; Jianxin, Z. Nanotechnology 2010, 21, 245701.

(5) Zhi, C.; Bando, Y.; Tang, C.; Kuwahara, H.; Golberg, D. Adv. Mater. 2009, 21, 2889.

(6) Tamor, M. A.; Vassell, W. C. Google Patents, 1996.

(7) Chen, Y.; Zou, J.; Campbell, S. J.; Le Caer, G. Appl. Phys. Lett. 2004, 84, 2430

(8) Kubota, Y.; Watanabe, K.; Tsuda, O.; Taniguchi, T. Science 2007, 317, 932.

(9) Britnell, L.; Gorbachev, R. V.; Jalil, R.; Belle, B. D.; Schedin, F.; Katsnelson, M. I.; Eaves, L.; Morozov, S. V.; Mayorov, A. S.; Peres, N. M. Nano Lett. 2012, 12, 1707.

(10) Liu, Z.; Gong, Y.; Zhou, W.; Ma, L.; Yu, J.; Idrobo, J. C.; Jung, J.; MacDonald, A. H.; Vajtai, R; Lou, J. Nat. Commun. 2013, 4.

(11) Li, L. H.; Cervenka, J.; Watanabe, K.; Taniguchi, T.; Chen, Y. ACS Nano 2014, 8, 1457.

(12) Kim, K. K.; Hsu, A.; Jia, X.; Kim, S. M.; Shi, Y.; Dresselhaus, M.; Palacios, T.; Kong, J. ACS Nano 2012, 6, 8583.

(13) Kim, K. K.; Hsu, A.; Jia, X.; Kim, S. M.; Shi, Y.; Hofmann, M.; Nezich, D.; Rodriguez-Nieva, J. F.; Dresselhaus, M.; Palacios, T.; Kong, J. Nano Lett. 2012, 12, 161.

(14) Yang, P. C.; Prater, J. T.; Liu, W.; Glass, J. T.; Davis, R. F. J. Electron. Mater. 2005, 34, 1558.

(15) Orofeo, C.; Suzuki, S.; Kageshima, H.; Hibino, H. Nano Res. 2013, 6, 335

(16) Kim, G.; Jang, A. R.; Jeong, H. Y.; Lee, Z.; Kang, D. J.; Shin, H. S. Nano Lett. 2013, 13, 1834. 
(17) Lu, G.; Wu, T.; Yuan, Q.; Wang, H.; Wang, H.; Ding, F.; Xie, X.; Jiang, M. Nat. Commun. 2015, 6, 6160.

(18) Wang, M.; Jang, S. K.; Jang, W.-J.; Kim, M.; Park, S.-Y.; Kim, S.W.; Kahng, S.-J.; Choi, J.-Y.; Ruoff, R. S.; Song, Y. J.; Lee, S. Adv. Mater. 2013, 25, 2746.

(19) Liu, B.; Tang, D. M.; Sun, C.; Liu, C.; Ren, W.; Li, F.; Yu, W. J.; Yin, L. C.; Zhang, L.; Jiang, C.; Cheng, H. M. J. Am. Chem. Soc. 2011, 133, 197.

(20) Chen, J.; Wen, Y.; Guo, Y.; Wu, B.; Huang, L.; Xue, Y.; Geng, D.; Wang, D.; Yu, G.; Liu, Y. J. Am. Chem. Soc. 2011, 133, 17548.

(21) Bhaviripudi, S.; Jia, X.; Dresselhaus, M. S.; Kong, J. Nano Lett. 2010, 10, 4128

(22) Tay, R. Y.; Griep, M. H.; Mallick, G.; Tsang, S. H.; Singh, R. S.; Tumlin, T.; Teo, E. H.; Karna, S. P. Nano Lett. 2014, 14, 839.

(23) Kuzuba, T.; Sato, Y.; Yamaoka, S.; Era, K. Phys. Rev. B: Condens. Matter Mater. Phys. 1978, 18, 4440.

(24) Gorbachev, R. V.; Riaz, I.; Nair, R. R.; Jalil, R.; Britnell, L.; Belle, B. D.; Hill, E. W.; Novoselov, K. S.; Watanabe, K.; Taniguchi, T.; Geim, A. K.; Blake, P. Small 2011, 7, 465.

(25) Shi, Y.; Hamsen, C.; Jia, X.; Kim, K. K.; Reina, A.; Hofmann, M.; Hsu, A. L.; Zhang, K.; Li, H.; Juang, Z.-Y.; Dresselhaus, M. S.; Li, L.-J.; Kong, J. Nano Lett. 2010, 10, 4134.

(26) Song, L.; Ci, L.; Lu, H.; Sorokin, P. B.; Jin, C.; Ni, J.; Kvashnin, A. G.; Kvashnin, D. G.; Lou, J.; Yakobson, B. I.; Ajayan, P. M. Nano Lett. 2010, 10, 3209.

(27) Petravic, M.; Peter, R.; Kavre, I.; Li, L. H.; Chen, Y.; Fan, L.-J.;

Yang, Y.-W. Phys. Chem. Chem. Phys. 2010, 12, 15349.

(28) Liu, D.; Lei, W.; Qin, S.; Chen, Y. Sci. Rep. 2014, 4, 4453.

(29) Tauc, J.; Grigorovici, R.; Vancu, A. Phys. Status Solidi B 1966, 15, 627.

(30) Blase, X.; Rubio, A.; Louie, S. G.; Cohen, M. L. Phys. Rev. B: Condens. Matter Mater. Phys. 1995, 51, 6868.

(31) Hoffman, D. M.; Doll, G. L.; Eklund, P. C. Phys. Rev. B: Condens. Matter Mater. Phys. 1984, 30, 6051.

(32) Behura, S.; Berry, V. ACS Nano 2015, 9, 2227.

(33) Sreeprasad, T. S.; Nguyen, P.; Kim, N.; Berry, V. Nano Lett. 2013, 13, 4434.

(34) Lee, G. H.; Yu, Y. J.; Cui, X.; Petrone, N.; Lee, C. H.; Choi, M. S.; Lee, D. Y.; Lee, C.; Yoo, W. J.; Watanabe, K.; Taniguchi, T.; Nuckolls, C.; Kim, P.; Hone, J. ACS Nano 2013, 7, 7931.

(35) Yin, Z.; Li, H.; Li, H.; Jiang, L.; Shi, Y.; Sun, Y.; Lu, G.; Zhang, Q.; Chen, X.; Zhang, H. ACS Nano 2012, 6, 74.

(36) Wang, S.; Wang, X.; Warner, J. H. ACS Nano 2015, 9, 5246.

(37) Okada, M.; Sawazaki, T.; Watanabe, K.; Taniguch, T.; Hibino, H.; Shinohara, H.; Kitaura, R. ACS Nano 2014, 8, 8273.

(38) Gong, Y.; Lin, J.; Wang, X.; Shi, G.; Lei, S.; Lin, Z.; Zou, X.; Ye, G.; Vajtai, R.; Yakobson, B. I.; Terrones, H.; Terrones, M.; Tay, B. K.; Lou, J.; Pantelides, S. T.; Liu, Z.; Zhou, W.; Ajayan, P. M. Nat. Mater. 2014, 13, 1135.

(39) Liang, L.; Meunier, V. Nanoscale 2014, 6, 5394.

(40) Lee, Y. H.; Zhang, X. Q.; Zhang, W.; Chang, M. T.; Lin, C. T.; Chang, K. D.; Yu, Y. C.; Wang, J. T. W.; Chang, C. S.; Li, L. J. Adv. Mater. 2012, 24, 2320.

(41) Mao, N.; Chen, Y.; Liu, D.; Zhang, J.; Xie, L. Small 2013, 9, 1312 .

(42) Lu, C.-P.; Li, G.; Mao, J.; Wang, L.-M.; Andrei, E. Y. Nano Lett. 2014, 14, 4628. 